\title{
Redução do dispêndio energético e excesso de peso corporal em adolescentes
}

\section{Energy expenditure reduction and overweight in adolescents}

Maria Fernanda Petroli FRUTUOSO ${ }^{1}$

Elizabeth Maria BISMARCK-NASR ${ }^{2}$

Ana Maria Dianezi GAMBARDELLA ${ }^{3}$

\section{R E S U M O}

A contemporânea redução no dispêndio energético apresenta-se como fator determinante da atual epidemia de obesidade observada em centros urbanos. Em adolescentes esta modificação intensifica-se quando associada a atividades passivas de lazer, como assistir televisão, brincar com jogos eletrônicos e usar a Internet. Este trabalho procurou analisar a inatividade física e a influência da televisão na prática alimentar de adolescentes, aspectos preditivos ao excesso de peso. Participaram deste estudo 155 indivíduos com média de 11,5 $\pm 1,43$ anos de idade. Os diagnósticos de sobrepeso e obesidade basearam-se no índice de massa corporal para adolescentes. Foram detectadas associações estatisticamente significativas entre apresentar sobrepeso e obesidade e praticar atividades passivas e consumir alimentos em frente à televisão para ambos os sexos. Estes achados mostraram que a inatividade física, associada ao aumento no consumo de alimentos energéticos enquanto assistem televisão ou em decorrência da influência de comerciais de produtos alimentícios por ela veiculados, representa um dos fatores determinantes para o desenvolvimento de peso corporal excessivo durante a adolescência.

Termos de indexação: adolescência, sobrepeso, prática alimentar, inatividade física, peso corporal, obesidade.

A B S T R A C T

The contemporary energy expenditure reduction is an important factor in the recent obesity epidemic observed in urban centers. In adolescents this change is more intense when it is associated with passive leisure activities,

\footnotetext{
1 Doutoranda, Faculdade de Saúde Pública, Universidade de São Paulo.

${ }^{2}$ Acadêmica, Curso de Nutrição, Faculdade de Saúde Pública, Universidade de São Paulo. Bolsista FAPESP, Processo 99/05461-7.

3 Departamento de Nutrição, Faculdade de Saúde Pública, Universidade de São Paulo. Av. Dr. Arnaldo 715, 01246-904, São Paulo, SP, Brasil. Correspondências para/Correspondence to: A.M.D. GAMBARDELLA. E-mail: gambarde@usp.br
} 
such as watching television, playing electronic games, playing and using Internet. The objective of this paper was to analyze the lack of physical activity and the influence of television in relation to the adolescents' feeding practices, aspects that can lead to overweight and obesity. This study included 155 individuals with a median age of $11.5 \pm 1.43$ years. The diagnoses of overweight and obesity were based on the body mass index for adolescents. Statistically significant correlations were observed betw een overweight/obesity and passive physical activities, as well as between overweight/obesity and the activity of eating while watching television, for both sexes. These findings showed that the physical inactivity, in conjunction with an increase in the energetic food consumption while watching television or due to the influence of television food commercials, represents a major factor in weight increasing during adolescence.

Index terms: adolescence, overweight, feeding practices, physical inactivity, body weight, obesity.

\section{N T R O D U ÇÃ O}

Nas últimas décadas vários estudos verificaram aumento na prevalência de obesidade em todas as faixas etárias e em vários países. Dentre suas causas, encontram-se fatores genéticos e ambientais ${ }^{1}$.

Dietz $(1998)^{2}$ considera a adolescência como um dos períodos críticos do desenvolvimento humano, pois as mudanças fisiológicas típicas desta fase podem aumentar o risco de estabelecimento da obesidade e de sua persistência futura. Estudos epidemiológicos ${ }^{3,4}$ mostraram que grande proporção de adultos obesos incorporaram essa característica durante a infância e adolescência.

A contemporânea redução no dispêndio energético apresenta-se como um dos fatores determinantes da atual epidemia de obesidade observada em grandes centros urbanos ${ }^{2}$. Em adolescentes, esta modificação intensifica-se quando associada a atividades passivas de lazer, como assistir televisão, brincar com jogos eletrônicos e usar a Internet.

Considerada de grande importância em saúde pública, a relação entre o número de horas diárias destinadas a assistir televisão e a obesidade infanto-juvenil encontra-se pioneiramente registrada por Dietz \& Gortmaker (1985)5. Segundo Robinson $(1998)^{6}$, dois mecanismos responsabilizam-se por esta relação: a diminuição do dispêndio energético, devido à redução da atividade física, e o aumento da ingestão ener- gética em frente à televisão ou decorrente dos anúncios por ela veiculados.

Segundo Wong et al. (1992)7, o tempo excessivo dedicado a assistir televisão mostra-se como um sinal mundial para identificação de crianças e adolescentes inseridos em estilos de vida que valorizam inadequados hábitos alimentares e inatividade física. Esta afirmação procede, pois pesquisas realizadas com adolescentes revelaram associação entre baixo consumo de frutas e hortaliças com elevada audiência de $\mathrm{TV}$, bem como ingestão insuficiente de vitamina C e distribuição inadequada de macronutrientes ${ }^{8}$.

Resultados obtidos por Jeferry \& French $(1998)^{9}$, referentes à população adulta feminina, condizem com a hipótese de que atitudes como assistir TV e consumir lanches rápidos com elevado conteúdo em gordura poderiam ter colaborado para o aumento da prevalência de obesidade nos EUA. Wong et al. (1992) ${ }^{7}$, analisando indivíduos norte-americanos entre 2 e 20 anos de idade, encontraram maior interesse por TV entre os mais velhos e, além disso, forte influência desta na elevação dos níveis séricos de colesterol.

Story \& Faulkner $(1990)^{10}$, observando os anúncios veiculados na TV norte-americana relacionados à alimentação, transmitidos entre 20 e 23 horas, identificaram que $60 \%$ destes referiam-se a refrigerantes e outros produtos alimentícios açucarados, com baixa qualidade nutricional. Em pesquisa realizada por Doyle \& Feldman (1997) ${ }^{11}$, 
$83 \%$ dos adolescentes residentes na Região Norte do Brasil apontaram a televisão como responsável por suas preferências alimentares.

Como estudos epidemiológicos mostraram que o sobrepeso na adolescência é fator preditivo da obesidade e/ou de diversas outras morbidades no adulto ${ }^{2,3}$, este trabalho procurou analisar a inatividade física e a influência da TV na prática alimentar de adolescentes, aspectos preditivos ao excesso de peso nesta fase do desenvolvimento humano.

\section{CASUÍSTICA E MÉTODOS}

Esta pesquisa foi realizada com adolescentes, freqüentadores de um centro de juventude do município de São Paulo; uma instituição filantrópica responsável pelo atendimento a crianças e adolescentes, entre 7 e 14 anos de idade, de baixo nível socioeconômico, onde estes permanecem meio período do dia e recebem duas refeições diárias (almoço e café da manhã/lanche da tarde), além de terem a oportunidade de desenvolver outras atividades profissionalizantes (informática, datilografia e educação artística) e recreativas (atividade física e teatro).

Os diagnósticos de sobrepeso e obesidade basearam-se no critério de classificação do Índice de Massa Corporal (IMC) para adolescentes, segundo sexo e idade, proposto por Cole et al. $(2000)^{12}$. Os pontos de corte foram obtidos com base em estudos transversais, de representação populacional, realizados no Brasil, Inglaterra, Estados Unidos, Holanda, Singapura e Hong Kong. Foram considerados os valores de IMC iguais a 25 para diagnóstico de sobrepeso e a 30 para diagnóstico de obesidade aos 18 anos de idade; em seguida, tais pontos de corte foram extrapolados para as demais idades (Tabela 1). Desta forma, a determinação dos pontos de corte diferiu de outros padrões estabelecidos com base em distribuições percentilares de uma única população de referência.
Tabela 1. Pontos de corte para diagnóstico de sobrepeso e obesidade segundo sexo e idade.

\begin{tabular}{lcclcc}
\hline \multirow{2}{*}{ Idade } & \multicolumn{2}{c}{ Sobrepeso } & & \multicolumn{2}{c}{ Obesidade } \\
\cline { 2 - 3 } \cline { 5 - 6 } & Meninos & Meninas & & Meninos & M eninas \\
\hline 10 & 19,84 & 19,86 & & 24,00 & 24,11 \\
11 & 20,55 & 20,74 & & 25,10 & 25,42 \\
12 & 21,22 & 21,68 & & 26,02 & 26,67 \\
13 & 21,91 & 22,58 & & 26,84 & 27,76 \\
14 & 22,62 & 23,34 & & 27,63 & 28,57 \\
\hline
\end{tabular}

Fonte: Cole et al. $(2000)^{12}$.

A inatividade física (ou prática de atividades passivas) foi calculada segundo a soma do tempo semanal dedicado a televisão, jogos eletrônicos e computador. Tal procedimento, adaptado de Tucker $(1986)^{13}$, classifica a inatividade física em três estágios: nível I - menos de 14 horas semanais; nível II - entre 14 e 28 horas; nível III - mais de 28 horas.

A atividade física semanal foi classificada de acordo com o dispêndio energético em leve, moderada e intensa, seguindo o padrão estabelecido em 1985 pela Organização Mundial da Saúde ${ }^{14}$

Para a análise dos dados utilizou-se a média, como medida de tendência central, e o desvio-padrão, para descrever a variabilidade dos resultados obtidos. A plicou-se o teste qui-quadrado $\left(\chi^{2}\right)$, no sentido de detectar associações entre as variáveis, e teste " $\mathrm{t}$ " de Student, para comparação entre as médias dos diferentes grupos $(\alpha=0,05)$.

Este trabalho apresenta-se de acordo com a Resolução 196/96 do Conselho Nacional de Saúde do M inistério da Saúde.

\section{RESULTA D O S}

Participaram deste estudo 155 adolescentes com 11,5 $\pm 1,43$ anos, em média, sendo $62 \%$ pertencentes ao sexo masculino e $38 \%$ ao feminino. A média de idade para as meninas foi $11,41 \pm 1,35$ e para os meninos, $11,63 \pm 1,48$ $(p>0,05)$.

Foi detectado excesso de peso para proporção semelhante de meninos e meninas, 
sendo de $23,7 \%$ e $23,0 \%$, respectivamente. Contudo, dentre esses indivíduos, encontrou-se maior proporção de meninas $(85,7 \%)$ do que de meninos $(72,7 \%)$ com sobrepeso, e o contrário foi verificado em relação à obesidade, $27,3 \%$ entre os do sexo masculino e de $14,3 \%$ entre os do sexo feminino (Tabela 2). Não foi observada associação entre sobrepeso, obesidade e sexo.

A prática de exercícios físicos entre os meninos atingiu a média de 3,6 $\pm 1,2$ dias por semana e entre as meninas, $2,4 \pm 1,5$ dias $(p<0,05)$. A maioria dos garotos $(93,5 \%)$ afirmou praticar atividade física intensa durante a semana, enquanto as adolescentes mostraram-se interessadas em atividade física moderada $(51,0 \%$ ) e intensa $(49,0 \%)$ (Tabela 3). Entre as atividades intensas mais citadas, destacaram-se futebol e capoeira, enquanto a prática de vôlei representou a maioria dos exercícios moderados. As demais modalidades de exercícios citados foram jogar basquete, brincar de queimada, praticar natação, andar de bicicleta e pular corda. Nos finais de semana, os entrevistados mostraram-se menos ativos que durante a semana.

No tocante à inatividade física, $38,7 \%$ dos adolescentes enquadraram-se no nível III, sendo $44,0 \%$ destes pertencentes ao sexo feminino e $35,4 \%$ ao masculino. No nível II situaram-se $37,5 \%$ e $39,0 \%$ dos meninos e meninas, respectivamente (Tabela 4).

Verificou-se que $50,5 \%$ das horas semanais de 39,0\% dos adolescentes foram dispendidas assistindo TV, divertindo-se com jogos eletrônicos e/ou computador e dormindo. Somente assistir televisão representou, em média, 13,0\% do tempo livre destes jovens.

Quanto à influência da televisão na prática alimentar, $78,1 \%$ dos meninos e $72,4 \%$ das meninas consumiam alimentos enquanto assistiam televisão, destacando-se como os mais citados biscoitos, refrigerantes, salgadinhos industrializados, pipoca e pães. Poucos adolescentes ainda referiram a ingestão de frutas, leite, sanduíches, doces e chocolates.

Tabela 2. Distribuição dos adolescentes segundo sexo e inadequação dos valores de IMC*.

\begin{tabular}{|c|c|c|c|c|c|c|c|}
\hline \multirow{2}{*}{ Inadequação do IMC* } & \multicolumn{2}{|c|}{ Meninos } & \multicolumn{2}{|c|}{ Meninas } & \multicolumn{2}{|c|}{ Total } & \multirow[b]{2}{*}{ Valor de $p$} \\
\hline & $\mathrm{n}$ & $\%$ & $\mathrm{n}$ & $\%$ & $\mathrm{n}$ & $\%$ & \\
\hline Sobrepeso & 16 & 72,7 & 12 & 85,7 & 28 & 77,7 & 0,93 \\
\hline Obesidade & 6 & 27,3 & 2 & 14,3 & 8 & 22,3 & 0,68 \\
\hline Total & 22 & 100,0 & 14 & 100,0 & 36 & 100,0 & \\
\hline
\end{tabular}

${ }^{(*)}$ IMC $=$ Índice de Massa Corporal.

Tabela 3. Distribuição (\%) dos adolescentes segundo sexo e intensidade da atividade física praticada semanalmente.

\begin{tabular}{|c|c|c|c|c|}
\hline \multirow{2}{*}{ Intensidade da atividade física } & \multicolumn{2}{|c|}{ Dias escolares } & \multicolumn{2}{|c|}{ Final de semana } \\
\hline & Meninos $(n=93)$ & Meninas $(n=49)$ & Meninos $(n=79)$ & Meninas $(n=40)$ \\
\hline Leve & 0,0 & 0,0 & 32,3 & 79,3 \\
\hline Monderada & 6,5 & 51,0 & 12,5 & 19,0 \\
\hline Intensa & 93,5 & 49,0 & 55,2 & 1,7 \\
\hline
\end{tabular}

Tabela 4. Distribuição dos adolescentes segundo sexo e intensidade de inatividade física.

\begin{tabular}{|c|c|c|c|c|c|c|}
\hline \multirow{2}{*}{ Intensidade física (horas/semana) } & \multicolumn{2}{|c|}{ Meninos } & \multicolumn{2}{|c|}{ Meninas } & \multicolumn{2}{|c|}{ Total } \\
\hline & $\mathrm{n}$ & $\%$ & $n$ & $\%$ & $\mathrm{n}$ & $\%$ \\
\hline Nível I (< 14) & 26 & 27,1 & 10 & 17,0 & 36 & 23,3 \\
\hline Nível II (14 - 28) & 36 & 37,5 & 23 & 39,0 & 59 & 38,0 \\
\hline Nível III (> 28) & 34 & 35,4 & 26 & 44,0 & 60 & 38,7 \\
\hline Total & 96 & 100,0 & 59 & 100,0 & 155 & 100,0 \\
\hline
\end{tabular}


A família foi apontada como a principal influência na escolha dos alimentos consumidos por $79,2 \%$ das respostas masculinas e $84,5 \%$ das femininas. As propagandas veiculadas pela televisão apresentaram interferência nesta decisão para $7,3 \%$ dos meninos e $8,6 \%$ das meninas. 0 restante dos adolescentes considerou os amigos como responsáveis por esta opção.

$\mathrm{Na}$ análise comparativa dos dados, foram detectadas associações estatisticamente significativas entre apresentar sobrepeso e obesidade e praticar atividades passivas de nível II para os meninos $\left(\chi^{2}=4,17 ; p=0,04\right)$ e de nível III para as meninas $\left(\chi^{2}=4,89 ; p=0,02\right)$ e entre apresentar sobrepeso e obesidade e consumir alimentos em frente à TV para ambos os sexos (meninos $\chi^{2}=56,34 ; p=0,00$; e meninas $\left.\chi^{2}=25,86 ; p=0,00\right)$.

\section{DIS C U SS Ã O}

Apesar de os valores referentes à prevalência de excesso de peso encontrados, em torno de $20 \%$ para ambos os sexos, não se aproximarem do verificado em países como Canadá ${ }^{15}$ e Estados Unidos ${ }^{16}$, onde mais de $30 \%$ dos adolescentes analisados apresentaram esta característica, este resultado, bem como o observado em jovens cearenses ${ }^{17}$, ilustra 0 sobrepeso na adolescência como uma questão importante, que necessita de medidas preventivas, com a finalidade de não alcançar os valores exibidos por outros países.

Acredita-se que o Centro de Juventude, freqüentado pelos adolescentes estudados, incentive a prática de atividade física, pois eles se revelaram mais ativos durante a semana em relação ao final de semana.

De um modo geral, as camadas sociais mais carentes mostram-se mais sedentárias, devido ao reduzido número de espaços públicos destinados à promoção da prática de exercícios físicos e em virtude da dificuldade de deslocamento em grandes centros urbanos. De fato, estudos de M cM urray et al. $(2000)^{16}$ e Pimenta et al. $(1999)^{18}$ realizados com adolescentes de diferentes níveis socioeconômicos, detectaram maior hábito sedentário entre aqueles de baixo nível socioeconômico.

A preferência das meninas por atividades físicas menos intensas, verificada no presente trabalho, vai ao encontro dos relatos de M cM urray et al. $(2000)^{16}$ e Nuzzo (1998) ${ }^{19}$.

Fonseca et al. $(1998)^{20}$, estudando adolescentes residentes em Niterói, RJ, verificaram que $31,6 \%$ praticavam exercício físico entre 4 e 5 dias por semana, correspondendo a $29,5 \%$ dos meninos e $33,7 \%$ das meninas, enquanto proporções semelhantes $(36,8 \%$ dos meninos e $26,5 \%$ das meninas) informaram assistir televisão durante mais de 3 horas diárias.

Ao contrário do observado neste estudo, M cM urray et al. $(2000)^{16}$ relataram semelhanças na prática de assistir televisão durante toda a semana (inclusive nos finais de semana) em adolescentes norte-americanos. Por outro lado, jovens franceses mostraram aumento no número de horas destinadas aos passatempos sedentários durante o final de semana ${ }^{21}$.

O consumo de alimentos ricos em monossacarídeos e lipídios enquanto assistiam TV, informado por $76 \%$ dos adolescentes, pode indicar inadequada prática alimentar, permitindo, a longo prazo, o desenvolvimento de sobrepeso. Este achado coincide com o verificado em um grupo de adolescentes cearenses ${ }^{17}$ e em crianças e adolescentes espanhóis ${ }^{22}$.

A grande disponibilidade no mercado de produtos alimentícios ricos em gordura, de baixo custo e saborosos, é apontada como um dos fatores responsáveis pelo aumento da prevalência de obesidade nos EUA ${ }^{23}$, Brasil ${ }^{24}$ e América Latina ${ }^{25}$.

A família influencia a escolha dos alimentos e/ou preparações consumidos pelos indivíduos avaliados, enquanto as campanhas publicitárias veiculadas pela televisão interferem na decisão dos entrevistados com menor importância. 
Resultados semelhantes foram observados em pesquisas com adolescentes paulistas ${ }^{19} \mathrm{e}$ australianos ${ }^{26}$, nas quais a família também representou o fator com maior influência na prática alimentar dos grupos estudados.

Ao contrário do verificado neste estudo, Ortega et al.(1996) ${ }^{8}$, analisando adolescentes espanhóis, detectaram nestes jovens maior susceptibilidade às mensagens recebidas de comerciais televisivos. Vale ressaltar que a maioria destes comerciais é veiculada durante os períodos destinados à programação infanto-juvenil e refere-se a produtos alimentícios de baixo conteúdo nutricional27.

0 consumo alimentar enquanto assistem TV e o tempo excessivo dedicado a esta atividade mostraram-se como fatores preditivos à obesidade nos adolescentes, uma vez que foram detectadas associações estatisticamente significativas entre apresentar sobrepeso e obesidade e atividades passivas de nível II, para os meninos, e de nível III, para as meninas, e entre apresentar sobrepeso e obesidade e consumir alimentos em frente à $\mathrm{TV}$, para ambos os sexos. Achados semelhantes foram relatados em outros estudos ${ }^{28,29}$.

\section{O N C L U S Ã O}

Os resultados observados no presente estudo sugerem que a inatividade física, associada ao aumento na ingestão de alimentos ricos em monossacarídeos e lipídios em frente à televisão, representa um dos fatores determinantes para o desenvolvimento de sobrepeso entre os adolescentes estudados. Deve-se direcionar atenção especial às meninas, pois elas apresentaram maior prevalência de risco de sobrepeso, além da diminuição na prática de atividade física e acréscimo no número de horas semanais destinadas às atividades passivas de lazer.

\section{REFER Ê N C I A S}

1. World Health Organization. Obesity: preventing and managing the global epidemic. Geneva; 1997.
2. Dietz WH. Health consequences of obesity in youth: childhood predictors of adult disease. Pediatrics 1998; 101:518-25.

3. Must $A$, Jacques PF, Dallal GE, Bajema CJ, Dietz $W H$. Long-term morbidity and mortality of overweight adolescents. A follow-up of the Haward Growth Study of 1922 to 1935. N Engl J Med 1992; 327(19):1350-55.

4. Bouchard C. Obesity in adulthood: the importance of childhood and parental obesity. N Engl J Med 1997; 73(13):926-27.

5. Dietz WH, Gortmaker SL. Do we fatten our children at the television set? Obesity and television viewing in children and adolescents. Pediatrics 1985; 75:807-12.

6. Robinson TN. Does television cause childhood obesity? J Am Med Assoc 1998; 279(12):959-60.

7. Wong ND, Hei TK, Qaqundah PY, Davidson DM, Bassin SL, Gold KV. Television viewing and pediatric hypercholesterolemia. Pediatrics 1992; 90(1):75-9.

8. Ortega RM, Requejo AM, Andrés P, López-Sobaler AM, Redondo R, González-Fernández M . Influence of the time spent watching television on the dietary habits, energy intake and nutrient intake of a group of Spanish adolescents. Nutr Res 1996; 16:1467-70.

9. Jeffery RW, French SA. Epidemic obesity in the United States: are fast foods and television viewing contributing? Am J Public Health 1998; 88:277-80.

10. Story M, Faulkner P. The prime time diet: a content analysis of eating behavior and food messages in television program content and commercials. Am J Public Health 1990; 80:738-40.

11. Doyle El, Feldman RHL. Factors affecting nutrition behavior among middle-class adolescents in urban area of Northern region of Brazil. Rev Saúde Pública 1997; 31(4):342-50.

12. Cole TJ, Bellizi MC, Flegal KM, Dietz WH. Establishing a standard definition for child overweight and obesity worldwide: international survey. Br Med J 2000; 320:1240-3. 
13. Tucker LA. The relationship of television viewing to physical fitness and obesity. Adolescence 1986; 21(84):797-806.

14. Organização Mundial da Saúde. Necessidades de energia e proteína. Geneva; 1985. (Série de Relatos Técnicos, n.724).

15. O' Loughlin J, Paradis G, Renaud L, Meshefedjian $G$, Gray-Donald K. Prevalence and correlates of overweight among elementary schoolchildren in multiethnic, low income, inner-city neighbourhoods in Montreal, Canada. Ann Epidemiol 1998; 8(7):422-32.

16. M cM urray RG, Harrell JS, Deng S, Bradley CB, Cox LM, Bangdiwala SI. The influence of physical activity, socioeconomic status and ethnicity on the weight status of adolescents. Obesity Res 2000; 8(2):130-9.

17. Passamai M PB. Estado nutricional, conhecimentos de nutrição em saúde e práticas alimentares de adolescentes de uma rede particular de ensino em Fortaleza-Ceará [mestrado]. Fortaleza: Universidade Estadual do Ceará; 1999.

18. Pimenta CD, Gambardella AMD, Cardoso MRA, Bismarck-Nasr EM . Relação entre a taxa de gordura corporal e assistir televisão em adolescentes de diferentes níveis socioeconômicos. In: Anais do $32^{\circ}$ Simpósio Internacional de Ciências do Esporte; 1999 outubro 7-10; São Paulo, Brasil. São Paulo: CELAFISCS; 1999. p.154.

19. Nuzzo L. Avaliação do estado nutricional de adolescentes de uma instituição particular de ensino. São Paulo [mestrado]. São Paulo: Faculdade de Saúde Pública, Universidade de São Paulo; 1998.

20. Fonseca VM, Sichieri R, Veiga GV. Fatores associados à obesidade em adolescentes. Rev Saúde Pública 1998; 32(6):541-9.

21. Frelut ML. De l'obésité de l'enfant à obésité de l'adulte. Cahiers Nutr Diététique 2001; 36(2): 123-7.
22. Ruano IR, PUJOL MES. Hábitos de vida en una población escolar de M ataró (Barcelona) asociados al número de veces diarias que ve televisión y al consumo de azúcares. Rev Esp de Salud Pública 1997; 71(5):1135-37.

23. Kuczmarski RJ, Flegal KM, Campbell SM, Johnson $\mathrm{CL}$. Increasing prevalence of overweight among US adults. The national health and nutrition examination surveys, 1960 to 1991. J Am Med Assoc 1994; 272:205-11.

24. Sichieri R, Coitinho DC, Leão MM, Recine E, Everhart JE. High temporal, geographic, and income variation in body mass index among adults in Brazil. Am J Public Health 1994; 84(5):793-98.

25. Peña M, Bacallao, J. La obesidad en la pobreza: un problema emergente en las Américas. In: Organización Panamericana de la Salud. La obesidad en la pobreza: un nuevo reto para la salud pública. Washington DC: OPS; 2000. (Publicación Científica, n.576).

26. Woodward DR, Boon JA, Cumming FJ, Ball PJ, Williams HM, Hornsby H. Adolescents' reported usage of selected foods in relation to their perceptions and social norms for those foods. Appetite 1996; 27:109-17.

27. Grazini J, Amâncio OM S. Analogia entre comerciais e hábito alimentar de adolescentes. [Documento online] 1997. [citado 4 Maio 1999]. Disponível em: URL: http:// www.e-gastroped.com.br/dez97/ 602097.htm

28. Andersen RE, Crespo CJ, Bartlett SJ, Cheskin LJ, Pratt M. Relationship of physical activity and television watching with body weight and level of fatness among children. J Am Med Assoc 1998; 279(12):938-42.

29. Gambardella AMD, Bismarck-Nasr EM. Televisão e predisposição à obesidade em adolescentes. Rev Paul Pediatr 2000; 18(1):18-21.

Recebido para publicação em 5 de março de 2001 e aceito em 4 de setembro de 2002. 